\title{
Evaluation of the effectiveness of serum aromatase activity and tissue polypeptide specific antigen in the diagnosis of breast cancer and prediction of lymph node metastatic status
}

\author{
Taha I Hewala, a MD; Gehan M Shehata,b MD; Mahmoud A Hemida,c MD; \\ Nadia A Abd El-Moneim,d MD
}

a) Departments of Radiation Sciences

b) Biomedical Informatics and Medical Statistics

c)Experimental and Clinical Surgery

d)Cancer Management and Research

Medical Research Institute, Alexandria University, Egypt.

Co"espondence:e-mail: tahahewala@hotmail.com

\begin{abstract}
Aim: The aim of this study was to evaluate the effectiveness of serum tissue polypeptide specific antigen (TPS) and aromatase activity in the detection of primary breast cancer and prediction of lymph node metastasis.

Subjects and methods: Serum samples were obtained from 35 postmenopausal breast cancer females before surgery and 35 normal healthy volunteers. The clinicopathological data of patients were obtained from their pathological reports. Determination of serum TPS was done using a ready-for-use ELISA kit. Serum total estradiol and total testosterone were determined using ready-for-use RIA kits and the ratio of estradiol to testosterone (E2/T) was used as an indirect expression of aromatase activity. Univariate and multivariate logistic regression analysis were performed to evaluate the association between these biomarkers and breast cancer, as well as lymph node metastasis.

Results: For breast cancer prediction, either serum TPS or aromatase activity can be used with significant areas under the ROC curves of $97 \%$ \& $91.8 \%$, respectively. For lymph node metastasis prediction, serum aromatase activity alone had the best predictive value, with a significant area under the ROC curve of $80.3 \%$. Multivariate logistic regression analysis in which aromatase activity and TPS were included in one model gives $74 \%$ adequacy in prediction of lymph node metastasis.

Conclusion: To predict breast cancer, either serum TPS or aromatase activity can be applied, while, for prediction of lymph node metastasis serum aromatase activity is recommended.

Key words: Aromatase activity, tissue polypeptide specific antigen, breast cancer.
\end{abstract}

Introduction:

Clinical examination, mammography, fine needle aspiration cytology and open surgical biopsy are established diagnostic methods in breast cancer and the fmal diagnosis of cancer should be based on histopathology.l In breast cancer, many tumor markers have been studied in the hope for finding a blood test for cancer diagnosis, but none of them has had such sensitivity and specificity that it could replace conventional diagnostic methods.2

A number of serum markers are used as indicators for breast cancer. Of these, the most widely used are CA 15-3 and CEA. However, lack of sensitivity and specificity preclude the use of these serum markers for the early detection of breast cancer. There is an urgent need for cancer biomarkers with more accurate diagnostic capability, particularly for earlystage cancer.3 Several clinical studies have been published on tissue polypeptide specific antigen (TPS) determination in women with breast cancer with sensitivity ranging from 31$78 \% .4,5$ 
Tissue polypeptide specific antigen (TPS) is related closely to human cytokeratin 18 , which is an essential part of the protein cytoskeleton of epithelial cells.6 As opposed to CA 15-3, which serum concentration provides data about tumor mass, serum TPS levels indicate proliferative activity of malignant tissue even if the mass is smalJ.7

Elevated concentrations of TPS have been detected in association with a wide variety of malignancies, including breast cancer.s

Human aromatase, a member of the cytochrome P450 superfamily, is a $58 \mathrm{kDa}$. It catalyzes a complex reaction sequence that results in the conversion of androgens (C19), namely testosterone and androstenedione, into estrogens (C18), namely estradiol, and estrone, respectively.9 In women of reproductive age, the ovaries express high levels of aromatase and they are the main source of estrogens. After menopause peripheral tissues including breast, lung, brain, and liver become sites of estrogen synthesis as a result of aromatization of circulating androgens.1O Aromatase expression is elevated in certain malignancies, such as breast and endometrial carcinomas.11

The aim of this study was the comparative evaluation of aromatase activity and TPS concentrations in sera from patients with breast cancer before surgery and normal healthy controls. The diagnostic values of the assayed serum parameters were compared using the Receiver Operating Characteristic (ROC) curve analysis. The correlation between biomarker levels and breast cancer histopathological characteristics was determined. We also investigated whether any of the biomarkers or their combined measurement could be used in the prediction of lymph node involvement. Univariate survival analysis of patients was assessed using the Kaplan Meier method.

Subjects and methods:

Sample size calculation:

Sample size was calculated using NCSS 2000 (Number Cruncher Statistical System) and PASS (Power Analysis and Sample Size) Program. According to the results obtained by Hang\&Rong-cheng for TPS,12 the minimum sample size required was 40 (20 for breast cancer patients group and 20 for control group) to achieve $80 \%$ power to detect a difference in means of 236 between both groups with estimated group standard deviations of 351 and 35 and with a significance level (alpha) of 0.05 using a two-sided sample t-test.13

Seventy postmenopausal females were enrolled in this retrospective case- control study. A female was considered postmenopausal if more than 12 months passed since her last menstrual period. Females were divided into two groups: Groupi (breast cancer patient's group):It included 35 female patients with breast invasive ductal carcinoma of clinical stages II and III 14 (recently detected, not receiving surgery or chemotherapy). Their mean age was $(51.33 \pm 2.12)$ years. Patients were recruited from the Departments of Experimental and Clinical Surgery and Cancer Management \& Research of the Medical Research Institute, Alexandria University in the period from October 2007 to May 2008. Groupll (control group): It included 35 normal healthy volunteers of comparable age $(52.80 \pm 3.50)$, menstrual cycle and socioeconomic status as patients.

This research protocol was approved by the ethical committee of Medical Research Institute. Each patient underwent full history recording, thorough clinical examination, routine laboratory investigations including complete blood count (CBC), mammography of breast and ultrasonography of abdomen and liver, radiological investigations including Xray chest, CT scan and bone scan when needed and fine needle aspiration cytology (FNAC) of breast mass to establish the pathological diagnosis in the patients.

Pathologic information was obtained from pathology reports. Collected data included tumor size and grade, lymph node involvement and status of estrogen receptor (ER) and progesterone receptor (PR). Breast cancer was clinicaly staged by the oncologist according to the tumor-nodes-metastasis (TNM) classification.14

All 35 breast cancer patients were subjected to surgery (Modified Radical Mastectomy), 15 then received adjuvant combination chemotherapy [5-Fluorouracil, Adriamycin and Cyclophosphamide (FAC)] 16 for 6 cycles. Breast cancer patients were evaluated after 3 
and 6 cycles of chemotherapy clinically, laboratory and radiologically to estimate the clinical response. Also, these patients were followed up till May 2011 for assessment of overall survival.

\section{Laboratory investigations:}

Blood samples were collected from normal healthy volunteers and breast cancer patients before surgery. Immediately after withdrawing, blood samples were allowed to coagulate and were centrifuged for 20 minutes at $3500 \mathrm{rpm}$. The separated serum samples were aliquoted, frozen at $-80{ }^{\circ} \mathrm{C}$, and stored until assay time. After thawing, each serum aliquot was assayed only once. Determination of serum levels of tissue polypeptide specific antigen (TPS), total testosterone and estradiol were carried out at Radiation Sciences Department, Medical Research Institute, Alexandria University.

Determination of serum Tissue Polypeptide Specific Antigen (TPS):

Serum TPS levels were determined using a ready-for-use ELISA kit (DRG diagnostics, Germany) according to the manufacturer's protocol. Briefly, 50 J.Ll of each calibrator and patient's serum sample were added into each well. $50 \mathrm{JJLl}$ of the diluted HRP-conjugated Anti-TPS solution to each well. Wells were shaken on a rack shaker $(600 \mathrm{rpm})$ for $2 \mathrm{hrs}$ at room temperature. Wells were decanted and washed with $6 \times 0.3 \mathrm{~m} 1$ diluted wash solution. $200 \mathrm{~J} . L l$ tetramethyl-benzidine (TMB) substrate solution were added per well and incubated in darkness for $20 \mathrm{~min}$. $50 \mathrm{~J} . \mathrm{Ll}$ of stop solution were added per well and the wells were mixed well. Absorbance of every well was read at $450 \mathrm{~nm}$. A standard curve was constructed from which the concentration of TPS in each serum sample was determined. The assay sensitivity was $6 \mathrm{U} / \mathrm{L}$ with no detectable cross reactivity to other tumor associated antigens that may be present in serum.

Determination of serum total testosterone:

Serum total testosterone levels were determined using a ready-for-use solid-phase radioimmunoassay kit (RIA, Siemens, USA) according to the manufacturer's protocol. Briefly, 50 J.Ll of each calibrator and patient's serum sample were pipetted into the test tubes.1.O $\mathrm{ml}$ of t25I-labeled total testosterone tracer was added to every tube. Tubes were mixed well and incubated for $3 \mathrm{hrs}$ at $37{ }^{\circ} \mathrm{C}$, and then tubes were decanted thoroughly. Bound radioactivity in each tube was counted for 1 minute in a gamma counter (Perkin Elmer, Finland). A calibration curve was constructed from which the concentration of total testosterone ( $\mathrm{ng} / \mathrm{dl})$ in each serum sample was determined. The assay sensitivity was $4 \mathrm{ng} / \mathrm{dl}$ with high specificity for testosterone.

Determination of serum total estradiol:

Serum total estradiol levels were determined using a ready-for-use solid- phase radioimmunoassay kit (RIA, Siemens, USA) according to the manufacturer's protocol. Briefly, $100 \mathrm{JLLl}$ of each calibrator and patienf s serum sample were added into the test tubes. $1.0 \mathrm{~m} 1$ of $125 \mathrm{I}$-labeled estradiol tracer was added to every tube. Tubes were mixed well and incubated for $3 \mathrm{hrs}$ at room temperature, and then tubes were decanted thoroughly. Bound radioactivity in each tube was counted for 1 minute in a gamma counter (Perkin Elmer, Finland). A standard curve was constructed from which the concentration of estradiol $(\mathrm{pg} / \mathrm{ml})$ in each serum sample was determined The assay sensitivity was $8 \mathrm{pg} / \mathrm{ml}$ with high specificity for estradiol.

Estimation of serum aromatase activity: Each serum value of estradiol $(\mathrm{pg} / \mathrm{ml})$ was multiplied by 10 to be converted into its corresponding ng/dl unit. According to several studies, aromatase activity in each serum sample from every patient and normal healthy volunteer was estimated indirectly as the ratio of the total estradiol ( $\mathrm{ng} / \mathrm{dl})$ level divided by total testosterone level (ng/dl); (E2/T).17-19

Statistical analysis:

A-Data processing:

Data processing had two major objectives namely clean data by performing a series of comprehensive checks and producing analytic results which involved the recoding of variables.

i. Recoding of variables: The quantitative variables were recoded using different cut 
off points defined by ROC analysis to include them in the analysis.

ii. Exploration of the quantitative data for normality distribution.

B- Data analysis included:

1- Kolmogrov- Smirnov test for data exploration.

2-Calculation of the median and range for the quantitative variables.

3- Mann Whitney test was performed to compare between the medians of the two groups.

4- Logistic regression analysis is used to assess the impact of interaction between two predictors as TPS and aromatase on the lymph node involvement in breast cancer patients.

5- ROC curve analysis was done to detect the area under the curve (overall accuracy) and different cut off points with the associated sensitivity and specificity.

6- Survival analysis was done including survival life tables, Kaplan Meier's curves and Log Rank test to compare between two factors regarding the survival. $\mathrm{P}$ value less than 0.05 was considered statistically significant.

\section{Results:}

1- The diagnostic value of semm TPS and aromatase activity:

Serum TPS level and aromatase activity were significantly elevated in the breast cancer patients group compared with the control group Table(l). Comparing the diagnostic values of serum TPS and aromatase activity using the receiver operating characteristic (ROC) curve analysis showed significant greater area under the curve for TPS (97\%) compared with that for aromatase activity $(91.8 \%)$. The sensitivity and specificity were $97.1 \%$ and $85.7 \%$ at cutoff $67.5 \mathrm{U} / \mathrm{L}$ for TPS and $86 \%$ and $89 \%$ at cutoff 0.09 for aromatase activity Figure(1) and Table(2).

Table (1): TPS and aromatase activityin the breast cancer patients and normal healthy control groups.

\begin{tabular}{|l|c|c|c|}
\hline \multicolumn{2}{|c|}{ Biomarker } & $\begin{array}{c}\text { Control group } \\
(\mathrm{n}=35)\end{array}$ & $\begin{array}{c}\text { Breast cancer patient's } \\
\text { group }(\mathrm{n}=35)\end{array}$ \\
\hline \multirow{2}{*}{ TPS (U/L) } & Median & 33.0000 & $143.0000 *$ \\
\cline { 2 - 4 } & Range & $(10.00-100.00)$ & $(60.00-243.00)$ \\
\hline \multirow{2}{*}{$\begin{array}{l}\text { Aromatase } \\
\text { activity } \\
(\mathrm{E} 2 / \mathrm{T})\end{array}$} & Median & 0.0667 & $0.1600^{*}$ \\
\cline { 2 - 4 } & Range & $(0.01-0.09)$ & $(0.00-1.10)$ \\
\hline
\end{tabular}

*:Significance was considered at $P$-value $<0.05$

$n$ : Number of subjects in each group 


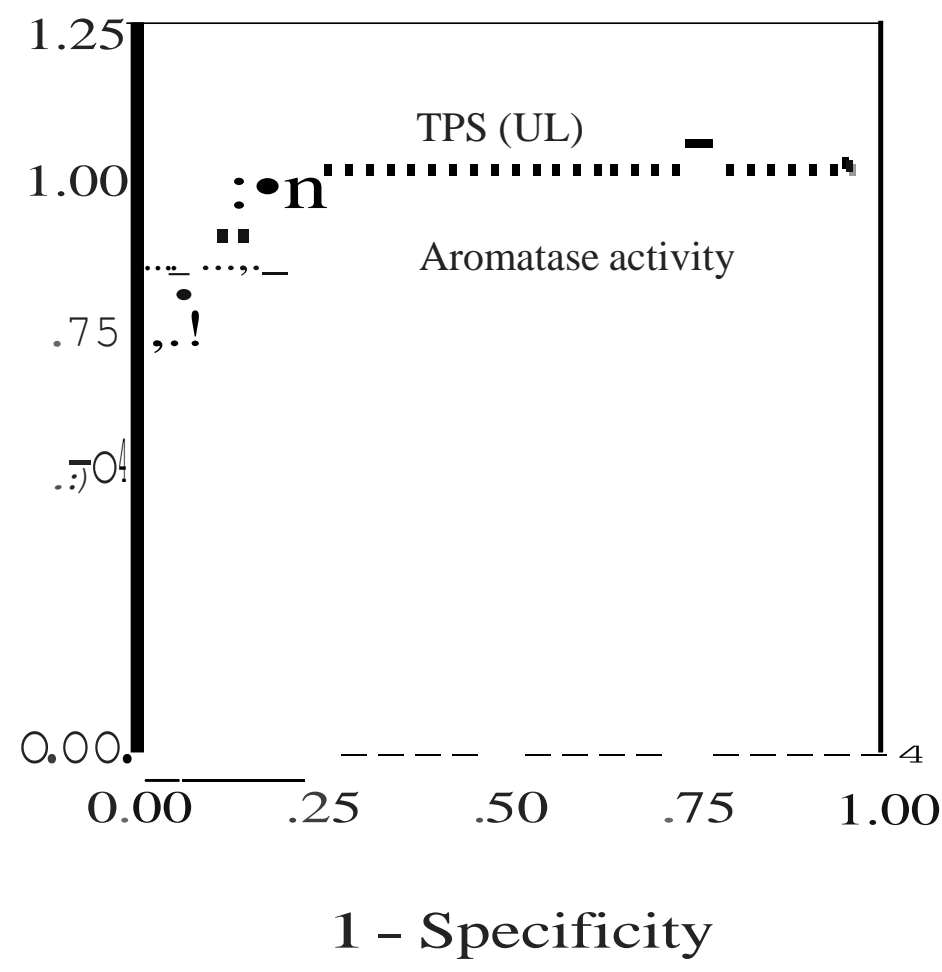

Figure (1): Graphical representation of the ROC curves for serum TPS and aromatase activity for detection of breast cancer.

Table (2): Area under the curve (AUC), cut-off, sensitivity and specificity for serum TPS $(U / L)$ and aromlltase activity for detection of breast cancer.

\begin{tabular}{|c|c|c|c|c|c|}
\hline Variables & $\begin{array}{c}\text { AUC } \\
\left(\frac{\circ}{0}\right)\end{array}$ & P-value & Cut-off & $\begin{array}{c}\text { Sensitivity } \\
(\%)\end{array}$ & $\begin{array}{c}\text { Specificity } \\
(\%)\end{array}$ \\
\hline $\begin{array}{c}\text { TPS } \\
(\mathrm{UIL})\end{array}$ & 97 & $0.02 *$ & 67.5 & 97.1 & 85.7 \\
\hline $\begin{array}{c}\text { Aromatase } \\
\text { activity } \\
(\mathrm{E} 2 / \mathrm{T})\end{array}$ & 91.8 & $0.01 *$ & 0.09 & 86 & 89 \\
\hline
\end{tabular}

*:Significance was considered at P-value $<0.05$

IT-Correlation of serum TPS and aromatase activity with breast cancer clinicopathological characteristics:

Serum TPS has no correlation with any of the clinicopathological characteristics ofbreast cancer patients. Wbilet aromatase activity was significantly correlated with lymph node involvement and tumor clinical stage Table(3). Comparison of the power of each serum marker to predict lymph node metastatic status using ROC curve yield a significant greater AUC for aromatase activity $(80.3 \%)$ than that of TPS $(38.9 \%)$. Also, from ROC curves, the sensitivity and specificity of aromatase activity were $76.2 \%$ \& $85.8 \%$ at cut-off 0.155 , while the sensitivity and specificity ofTPS were $71.4 \%$ \& $21.5 \%$ at cut-off 92.5 U/L Figure(1) and Table(4). Multivariate logistic regression analysis in which TPS and aromatase activity were included in one model gives AUC of $74 \%$.

Relationship between serum TPS and aromatase activity and patients' overall survival:

Kaplan-Meier analysis revealed that patients with elevated levels of serum TPS and 
aromatase activity had a survival nonsignificantly shorter than those who had lower levels of these biomarkers as shown in Table(S).

Table (3): Serum levels of TPS and aromatase activity [median (range)] in relation to clinicopathological characteristics of breast cancer patients.

\begin{tabular}{|c|c|c|}
\hline $\begin{array}{c}\text { Clinicopathological } \\
\text { characteristic }\end{array}$ & $\begin{array}{l}\text { TPS } \\
(\mathrm{U} / \mathrm{L})\end{array}$ & $\begin{array}{l}\text { Aromatase activity } \\
\qquad(\mathrm{E} 2 / \mathrm{T})\end{array}$ \\
\hline $\begin{array}{l}\text { Tumor size } \\
: \mathrm{S} 5 \mathrm{Cm} 3 \\
>5 \mathrm{Cm} 3\end{array}$ & $\begin{array}{l}173(60-243) \\
174(60-234)\end{array}$ & $\begin{array}{c}1.10(0.0-1.10) \\
0.82(0.10-0.92)\end{array}$ \\
\hline $\begin{array}{l}\text { Lymph node involvement } \\
\text { Negative } \\
\text { Positive }\end{array}$ & $\begin{array}{l}159(75-234) \\
183(60-243) \\
\end{array}$ & $\begin{array}{l}0.25(0.05-0.30) \\
1.10(0.00-1.10)^{*}\end{array}$ \\
\hline $\begin{array}{l}\text { Clinical stage } \\
\text { I I } \\
\text { III }\end{array}$ & $\begin{array}{l}127(90-217) \\
183(60-243)\end{array}$ & $\begin{array}{l}0.06(0.05-0.11) \\
1.10(0.00-1.10)^{*}\end{array}$ \\
\hline $\begin{array}{l}\text { Pathological grade } \\
\text { I\&II } \\
\text { III }\end{array}$ & $\begin{array}{l}183(60-243) \\
145(72-217)\end{array}$ & $\begin{array}{c}0.92(0.00-0.250) \\
1.00(0.10-1.10)\end{array}$ \\
\hline $\begin{array}{l}\text { ER } \\
\text { Negative } \\
\text { Positive }\end{array}$ & $\begin{array}{l}134(100-234) \\
183(60-243)\end{array}$ & $\begin{array}{l}0.28(0.10-0.38) \\
1.10(0.00-1.10)\end{array}$ \\
\hline $\begin{array}{l}\text { PR } \\
\text { Negative } \\
\text { Positive }\end{array}$ & $\begin{array}{l}174(60-234) \\
173(70-243)\end{array}$ & $\begin{array}{l}0.85(0.07-0.92) \\
1.10(0.00-1.10)\end{array}$ \\
\hline
\end{tabular}

*:Significance was considered at $P$-value $<0.05$

$E R \&$ PR: Estrogen and progesterone receptor 


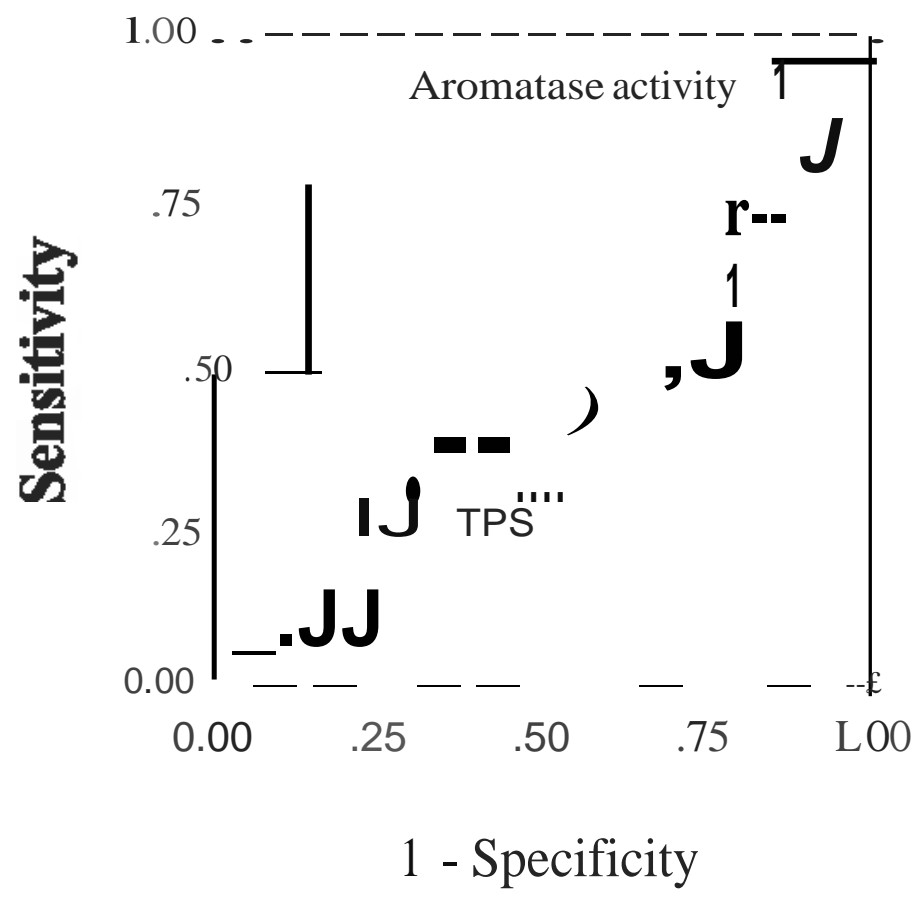

Figure (2): Graphical representation of the ROC curves for serum TPS (U/1) and aromatase activity for prediction of lymph node involvement in breast cancer patients group.

Table (4): Area under the curve (AUC), cut-off, sensitivity and specificity for serum TPS (UIL) tlld $t$ romatase tiCtivity for predicdon of lymph node involvement in breast cancer patients group.

\begin{tabular}{|c|c|c|c|c|c|}
\hline Variables & AUC(\%) & P-value & Cut-otT & $\begin{array}{c}\text { Sensitivity } \\
(\%)\end{array}$ & $\begin{array}{c}\text { Speeificity } \\
(\%)\end{array}$ \\
\hline $\begin{array}{c}\text { TPS } \\
(\mathrm{U} / \mathrm{L})\end{array}$ & 38.9 & $0.027 *$ & 92.5 & 71.4 & 21.5 \\
\hline $\begin{array}{c}\text { Aromatase } \\
\text { activity } \\
(\mathrm{E} 21 \mathrm{~T})\end{array}$ & 80.3 & $0.003 *$ & 0.155 & 76.2 & 85.8 \\
\hline
\end{tabular}

*:Significance was considered at P-value $<0.05$ 
Table (5): Correlation between serum TPS levels, aromatase activity and overall survival (months) among breast cancer patients group.

\begin{tabular}{|c|c|c|c|c|c|}
\hline & \multirow[t]{2}{*}{ Cut off values } & \multirow{2}{*}{$\begin{array}{c}\text { Survival } \\
\text { time } \\
\text { (months) } \\
\text { Mean } \pm \text { SE }\end{array}$} & \multicolumn{2}{|c|}{$\begin{array}{c}95 \% \text { Confidence } \\
\text { Interval }\end{array}$} & \multirow[b]{2}{*}{ P-Value } \\
\hline & & & $\begin{array}{l}\text { Lower } \\
\text { bound }\end{array}$ & $\begin{array}{l}\text { Upper } \\
\text { bound }\end{array}$ & \\
\hline \multirow[t]{2}{*}{$\begin{array}{l}\text { TPS } \\
\text { (UIL) }\end{array}$} & $\begin{array}{c}67.5 \\
\text { (Negative) }\end{array}$ & $38.33 \pm 6.01$ & 23.30 & 46.86 & $\mathrm{P}=0.941$ \\
\hline & $\begin{array}{c}>67.5 \\
\text { (Positive) }\end{array}$ & $35.08 \pm 2.35$ & 32.47 & 41.67 & \\
\hline $\begin{array}{c}\text { Aromatase } \\
\text { activity }\end{array}$ & $\begin{array}{c}<0.09 \\
\text { (Negative) }\end{array}$ & $41.20 \pm 1.32$ & 38.60 & 43.80 & $\mathrm{p}=0.605$ \\
\hline$(\mathrm{E} 2 / \mathrm{T})$ & $\begin{array}{c}.09 \\
\text { (Positive) }\end{array}$ & $35.36 \pm 3.28$ & 28.93 & 41.79 & \\
\hline
\end{tabular}

\section{Discussion:}

Early detection ofbreast cancer can improve cancer mortality, and early prediction of lymph node metastasis may facilitate the choice of operation type as well as use of adjuvant therapy. The use of serum markers has been well established in the screening and postoperative follow-up of different types of malignancies.20 The present most commonly used breast cancer serum markers, carcinoembryonic antigen (CEA) and breast cancer-specific cancer antigen 15.3 (CAl 5-3), have disadvantages including poor sensitivity, poor specificity and a lack of standard cut-off values for either primary breast cancer detection or metastatic status prediction.21 Several biomarkers have been proposed as potential new markers for breast cancer, including tissue polypeptide-specific antigen (TPS) which measures a specific epitope structure of soluble fragments ofhuman cytokeratin 18 , an acidictype intermediate filament protein detected in various types ofhuman cancer. 6

For TPS, at a cut-off level of 75-170 U L-1, the sensitivity and specificity for detection of primary breast cancer were reported to be $30-95 \%$ and $75-90 \%$, respectively.22,23 Kassim et al used TPS as a sensitive proliferative serum marker in screening for those at high risk of breast cancer.22 Hwa et al. showed that TPS was the best predictive marker for primary breast cancer at a cut- off value of $69.1 \mathrm{U} / \mathrm{L}$ with a sensitivity of $80 \%$ and specificity of $67 \% .21$

The findings of this study support serum TPS to be a diagnostic serum marker that differentiates breast cancer patients from normal healthy controls. At cut-off value of 67.5 U/L, TPS has sensitivity and specificity of97.1\% \& $85.7 \%$, respectively, with AUC of $97 \%$. Our results are in agreement with Kassim et al.22 who used serum TPS to differentiate Egyptian breast cancer females from females with bengin breast diseases. They reported that at a cut-off for TPS of $88 \mathrm{U} / \mathrm{L}$, the sensitivity is $95 \%$, and the specificity is $75 \%$ with AUC of $91 \%$. The greater cut-off value in Kassim et al. study 22 may be due to the use of patients with benign breast diseases instead of normal healthy volunteers. Also, different distribution of clinical stages of studied cases may influence the cut-off value.

Concerning the diagnostic value of serum aromatase activity, the present study showed the possibility of using this enzyme to differentiate between breast cancer females and normal healthy volunteers. At cut-off value of0.09, aromatase activity has sensitivity and specificity of $86 \%$ \& $89 \%$, respectively, with AUC of $91.8 \%$. By comparing the diagnostic values of serum TPS and aromatase activity using ROC curve, the AUC of TPS was found 
to be significantly greater thanthat of aromatase activity indicating that TPS is superior to aromatase activity in detecting breast cancer in postmenopausal females. To the best of our knowledge, no other previous or recent work studied the diagnostic role of this enzyme in the sera ofbreast cancer females.

The present study reveals that serum TPS is correlated with neither clinicopathological characteristics nor overall survival of breast cancer patients. Our study is compatible with the results reported by $\mathrm{Hu}$ et al.24

In our study, serum aromatase activity was significantly correlated with lymph node metastatic status and patient clinical stage. On the other hand, aromatase activity has no significant correlation with patients' overall survival. The absence of a significant correlation between serum TPS levels and aromatase activity with patients overall survival may be related to the small sample size used in the present study.

Univariate logistic regression analysis for prediction of lymph node metastasis showed that serum aromatase activity has the best predictive value for lymph node metastasis with AUC of $80.3 \%$, the sensitivity and specificity were $76 \%, 85.8 \%$, respectively, at a cut-off0.155.

The multivariate logistic regression model is a simple method for considering multiple continuous and/or categorical factors simultaneously. It can calculate the weighting on the individual prediction. A variety of markers, each of which had been reported to be elevated in patients with cancer of any type, were combined to see if multivariate analysis would yield information not available by the use of one marker alone.21 The developed multivariate logistic regression equation used in the present study presents the degree of contribution of each variable to the prediction.

To study the effect of combination of aromatase activity and TPS on the prediction of lymph node metastatic status, multivariate logistic regression analysis showed that combination of serum levels of aromatase activity and TPS resulted in $74 \%$ adequacy of predicting lymph node metastasis which means that this combination did not improve the accuracy of predicting lymph node metastasis achieved by aromatase activity alone. Therefore, to screen for breast cancer, either serum TPS or aromatase activity can be used, but in case of screening for lymph node metastasis in breast cancer patients, serum aromatase activity can be assayed with no need for assaying TPS in order to reduce the patient cost. This can meet the demands of different populations of different sizes.

In conclusion, serum TPS or aromatase activity can be used for detection of breast cancer, while only serum aromatase activity can be used as a predictive marker for lymph node metastasis.

\section{References:}

1- Gion M, Mione R, Gatti K: Is tissue polypeptide antigen still a useful tumor marker in breast carcinoma? Comparison with CA15-3 andMCA. Tumori 1990; 76: 360-364.

2- Jotti G S, Bombardieri E: Circulating tumor markers in breast cancer (review). Anticancer Res 1990; 10: 253-258.

3- Duffy MJ: Serum tumor markers in breast cancer: Are they of clinical value. Clin Chem 2006; 52: 345-351.

4- Ringash J: Canadian Task Force on Preventive Health Care. Preventive health care, 200 I update: Screening mammography among women aged 40---49 years at average risk of breast cancer. Canadian Medical Association Journal 2001; 164: 469-476.

5- Smigal C, Jemal A, WardE, et al: Trends in breast cancer by race and ethnicity: update 2006. CA: A Cancer Journal for Clinicians 2006; 56: 168-183.

6- Rydlander L, Ziegler E, Bergman T, et al: Molecular characterization of a tissuepolypeptide-specific-antigen epitope and its relationship to human cytokeratin 18 . Eur J Biochem 1996; 241: 309-314.

7- Bjorklund B, Einarsson R: TPS (tissue polypeptide specific antigen) in oncologic practice: A review with reference to 3000 cases of breast cancer. Tumordiagn Ther 1996; 17: 67.

8- D'Alessandro R, Roselli M, Ferroni P, et al: Serum tissue polypeptide specific antigen (TPS): A complementary tumor 
marker to CA15-3 in the management of breast cancer. Breast Cancer Research and Treahnent2001;68:9-19.

9- Simpson ER: Sources of estrogen and their importance. J Steroid Biochem Mol Biol 2003; 86: 225-230.

10-Gould RE, Garcia AA:Update on aromatase inhibitors in breast cancer. Curr Opin Obstet Gynecol 2006; 18: 41-46.

11-Shibayama T, Ueoka $\mathrm{H}$ and Nishii $\mathrm{K}$, et al: Complementary roles of pro-gastrinreleasing peptide (ProGRP) and neuron specific enolase (NSE) in diagnosis and prognosis of small-cell lung cancer (SCLC). Lung Cancer 2001; 32: 61-69.

12-Hang Z, Rong-cheng L: Diagnostic value of combined detection ofTPS, CA15.3 and CEA in breast cancer. J First Mil Med Univ 2005;25: 1293-1298.

13-Hintze J: NCSS (Number Cruncher Statistical System): Statistical Software, PASS (Power Analysis and Sample Size). Kaysville, Utah 2000.

14-Haskell CM, Lowitz BB, Casciato AD:

Breast cancer. In: Manual of clinical oncology. Casciato AD and Lowitz BB (Editors); Little and Brown Company, Boston, Toronto (Publishers); 2nd edn. 1985; p.150-165.

15-Rintoul RF: Operations on the breast. In: Farquhaerison's text book of operative surgery. Churchill living stone (Publisher); 7th edn. 1986; p. 270-281.

16-AbeloffMD, Lichter AS, Niederhuber JE, Pierce $U$ and Aziz DC: Breast In:Clinical Oncology. Abeloff MD, Armitage JO, Lieber AS, Niederhuber JE (Editors); Churchillivingstone Inc (Publisher); 1995; Chapter 73, p. 1617-1714.

17-Probst-Hensch NM, Ingles SA, Diep AT, Haile RW, Stanczyk FZ, Kolonel LN, Henderson BE: Aromatase and breast cancer susceptibility. Endocrine-Related Cancer 1999; 6: 165-173. 18-la Marca A, Morgante G, Palumbo M, Cianci A, Petraglia F, De Leo V: Aromatase activity in response to follicle stimulating hormone in women with polycystic ovary syndrome. Fertility and Sterility 2002; 78 : 1234-1239.

19-Amato MC, Verghi M, Nucera M, Galluzzo A, Giordano C: Low estradiol-totestosterone ratio is associated with oligoanovulatory cycles and atherogenic lipidic pattern in women with polycystic ovary syndrome. Gynecol Endocrinol2010; 7.

20-Ugrinska A, Bombardieri E, Stokkel MPM, Crippa F, Pauwels EKJ: Circulating tumor markers and nuclear medicine imaging modalities: Breast, prostate and ovarian cancer. Quarterly Journal of Nuclear Medicine 2002; 46: 88-104.

21-Hwa HL, Kuo WH, Chang LY, Wang MY, Tung TH, Chang KJ, Hsieh FJ: Prediction ofbreast cancer and lymph node metastatic status with tumour markers using logistic regression models. J Eval Clin Pract 2008; 14: 275-280.

22-Kassim SK, Fawzy H, El Rassad MM, Khalil FK, Khalifa A: Telomerase activity, and tissue polypeptide specific antigen (TPS) in Egyptian breast cancer patients. Clin Biochem 2001; 34: 499-504.

23-D'Alessandro R, Roselli M, Ferroni P, et al: Serum tissue polypeptide specific antigen (TPS): A complementary tumor marker to CA15-3 in the management of breast cancer. Breast Cancer Research and Treahnent 2001; 68: 9-19.

24-Hu XC, Day W, Jones B, Loo WT, Chow LW: Comparison of TPS with CEA and CA 15.3 in follow-up of Chinese breast cancer patients. Anticancer Res 2002; 22: 1865-1868. 